\title{
Opportunistic Cooperative Sensing for Indoor Complex Environment Monitoring
}

\author{
https://doi.org/10.3991/ijoe.v13i08.6709 \\ Yong Jin $\left.{ }^{\bowtie}\right)$, Zhenjiang Qian, Xiaoshuang Xing, Lu Shen \\ Changshu Institute of Technology, Changshu, China \\ jinyongecslg.cn
}

\begin{abstract}
Sensor failure have become a major bottleneck, which restricted the wide application of wireless sensor networks WSNs (Wireless Sensor Networks). In order to satisfy the needs of industrial production and daily living environment monitoring, it is important to improve the survivability of wireless sensor networks. This paper analyzed the damage types and causes of WSNs and the measurement methods of WSNs survivability. Then, based on the whole system model and sensor node circuit, we studied the fault detection method, which can improve the survivability of the scheme. Additionally, the robust guarantee scheme through hardware design and algorithm model was proposed. The performance results shown that the proposed scheme can realize the remote wireless communication services and prolong the network life cycle, so as to improve the survivability of WSNs. Through the combination of theoretical research and practical analysis system, the network survivability would be improved through soft and hard optimization combination strategy.
\end{abstract}

Keywords-Wireless sensor networks; invulnerability; indoor Complex environment monitoring; hardware design; robust guarantee

\section{Introduction}

The environmental monitoring system [1, 2] based on WSNs (Wireless Sensor Networks) is used to reduce the cost of the entire system, mostly using mass low-cost sensor nodes [3] for networking. Invulnerability of WSNs is the ability measurement metric of WSNs, which could be sustainable and stable to provide reliable services for the entire network system. In the case of random or deterministic failures [4] in the network communication, the system is able to complete the key services.

About the robust stability guarantees, the article [5] presented a new soft constrained model predictive control approach for tracking that provides stability guarantees even for unstable systems. The research of article [6] exploited the cubature rule in the unknown input observer structure to overcome nonlinear calculations in the presence of external disturbances for sensor fault detection purposes. In article [7], it is demonstrated that, under mild assumptions, the distributional robust optimization problems over Wasserstein balls can in fact be reformulated as finite convex programs---in many interesting cases even as tractable linear programs. A robust adaptive beamforming scheme based on two-component electromagnetic vector-sensor 
arrays was proposed by extending the well-known worst-case constraint into the quaternion domain in article [8]. The authors of article [9] focused on the problem of localization in mixed LOS (line-of-sight)/NLOS (non-line-of-sight) scenario and proposed a novel localization algorithm: Gaussian mixed model based non-metric Multidimensional.

About the robust system research, a self-powering wireless environment monitoring system was proposed by using renewable and cost-efficient soil energy [10]. The authors proposed the Civitavecchia coastal environment monitoring system, which is used to analyze the conflicts between coastal pressures and sensitivity areas in article [11]. The wireless sensor network, ZigBee technology and embedded technology were combined [12] to explore the implementation of the greenhouse environment monitoring system with low cost and high efficiency. The integrated environment management system was studied for better living conditions, which analyzed and managed the harmful environmental factors to make more pleasant environment in office, library or classroom [13].

On the basis of the above researches, we studied the fault detection method to provide the guarantee of the wide application of WSNs. Additionally, we proposed the survivability guarantee design scheme to satisfy the requirements of industrial production. The Quality of Services guarantee of the remote wireless communication was provided for designing the robust guarantee scheme. The scheme can prolong the network life cycle and improve the survivability of the Indoor environmental monitoring system.

The rest of this paper is organized as follows. Section 2 gives the algorithm model of opportunistic cooperative sensing robust guarantee. Section 3 gives the robust opportunistic guarantee architecture and implementation. Section 4 studied and evaluated the performance of the proposed scheme. Finally, the conclusion is made in Section 5. Section 6 shows the acknowledgement.

\section{Algorithm model of opportunistic cooperative sensing robust guarantee}

Based on hardware and software diversity of the sensor nodes (as shown in Figure 1), we designed the hard and soft optimization opportunistic cooperation guarantee architecture of the system robustness. The architecture can not only improve the survivability of the system but also enhance the robustness of communication networks. Specially, the architecture can prolong the overall life cycle effectively.

Here, the soft opportunistic control is completed by the system opportunistic control based on the algorithm model. The robust performance of system is improved by the redundancy perception of WSNs. In WSNs, the sensor nodes are low cost, usually with a lot of redundant nodes. When some nodes or links of WSNs are damaged, the data transmission may be abnormal. At this time, the link composed with the redundant nodes and the redundant nodes can play the role of fast backup communication node. This action can compensate for the loss of a node or link. This can improve the overall system reliability and robustness. In this paper, the algorithm of soft opportun- 
istic guarantee scheme is used to improve the robustness of WSNs by sensing the link redundancy and redundancy of cluster head for making full use of the redundant features of the WSNs.

Based on the hardware control design of sensor node, the hard opportunistic control can improve the system robust, by the network cooperative restructure and fault polling detection. Here, the network cooperative restructure improve the robust performance with adding the opportunistic relay nodes. This scheme can extend the distance of network communication and reduce the time of reestablishing the route due to impaired communication nodes or links, as well as reduce the power loss of intermediate node data transmission. By using distributed fault detection, through the data processing in the process of increasing the hardware design, the fault polling detection realize the fault warning. The data analyzed the collection data of the sensor in real time. The method judge the fault type with the numerical value. The fault types include: communication node hardware failure and warning of environmental parameters. This opportunistic design can not only improve the efficiency and robustness of fault detection, but also can know the location of the failure node in a timely manner.

The REED [14] (Robust Energy Efficient Distributed clustering) mainly solves the problem of cluster head node failure in communication link. However, this model does not solve the problem of common node communication reliability. The FLSSK [15] (Fault-tolerant Local Spanning Sub-graph) mainly solves the problem of communication failure between common nodes in the communication link. But it does not solve the reliability problem of cluster head node communication transmission. So, the fusion of REED and FLSSK based on redundancy was proposed as shown in Figure 2.

In this fusion model, there are $\mathrm{K}$ communication link between bottom layer sensors. Each sensors of the cluster would maintain contact with $\mathrm{N}$ cluster headsets. The improved algorithm model choose the optimal routing path between the sensor nodes and the cluster head node. When the remote communication link between the sensing node and cluster head or one node failure occurred, the data transmission would be implemented through multi path routing according to the characteristics of FLSSK model. If the cluster head node is damaged due to failure, the cluster head can be switched quickly according to the characteristics of the REED model, which can ensure the reliable data transmission. Thus, the improved algorithm model could further strengthen the reliability and robustness of data transmission in a communication link between the sensor nodes and the cluster head nodes, so as to improve the survivability of the system as a whole.

The limited sensor node communication radius and low bandwidth lead to the low data transmission capacity of long-distance. And the energy consumption of the longdistance transmission season is very high. So, the probability of intermediate node damage and failure is very large. Through the arrangement of some relay nodes in a communication network (Repeater) which could communicate with the common node, the relay node with its strong emission characteristics of power, communication distance and energy strong, can not only prolong the network communication distance, but also reduce the general perception of the node energy consumption to improve the overall performance of the network connectivity. 
Based on the FFRP (Full Fault-tolerance Relay node Placement), as shown in Figure 3 , there are at least two communicate link in each pair of nodes. Even if a node is damaged, which would not affect the overall connectivity of the network. This network reconfiguration design scheme could effectively enhance the network survivability.

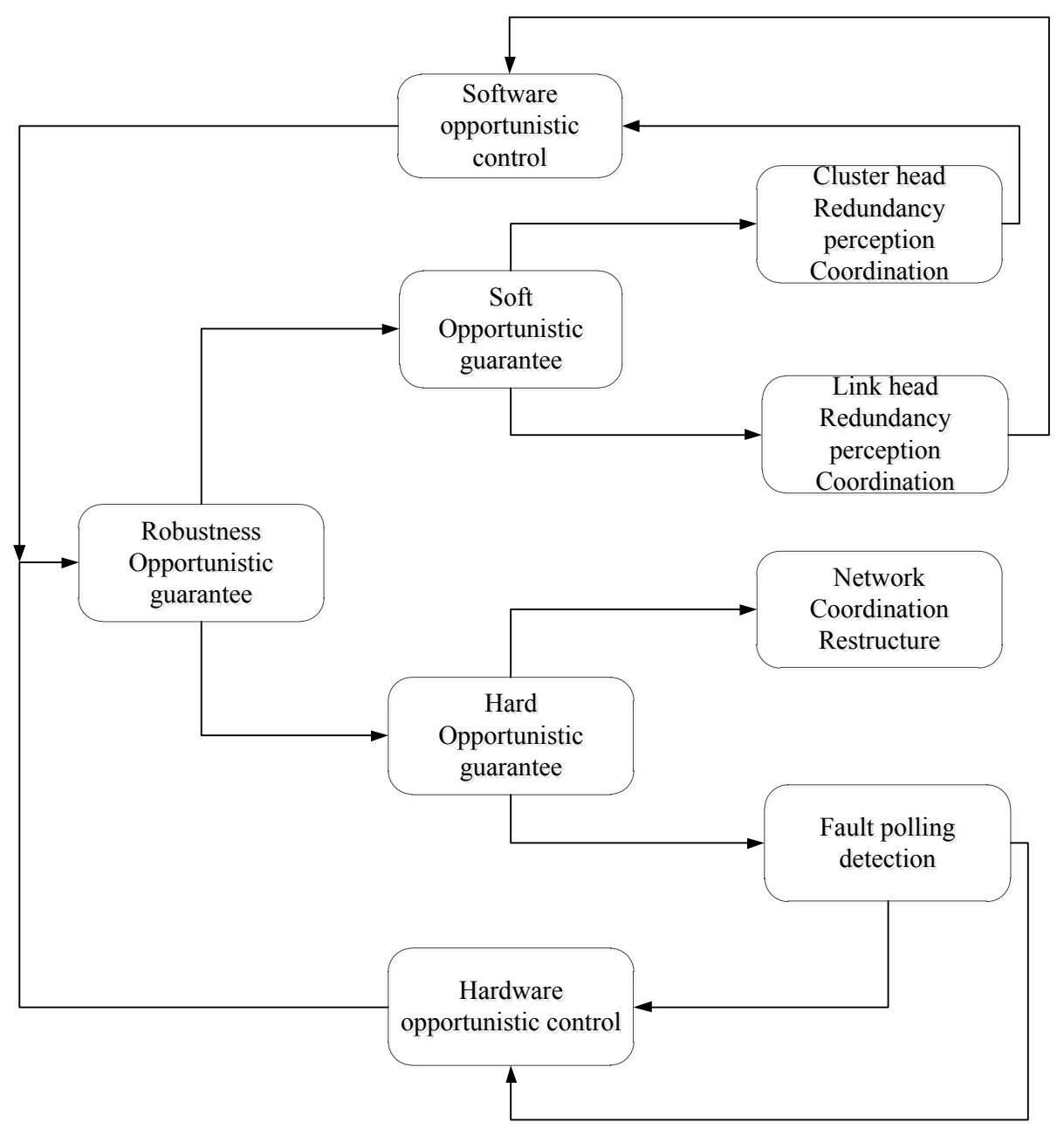

Fig. 1. The guarantee architecture 


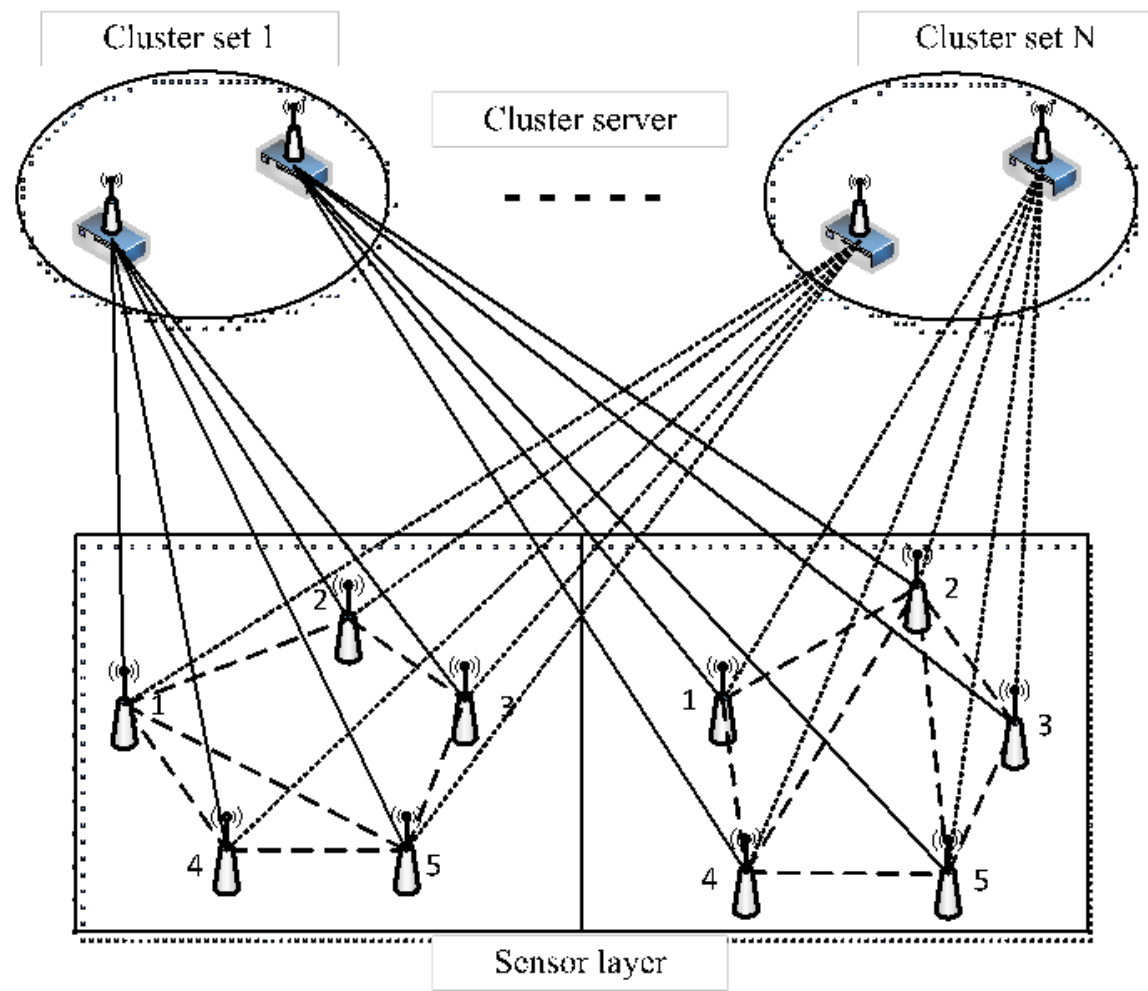

Fig. 2. the fusion algorithm model
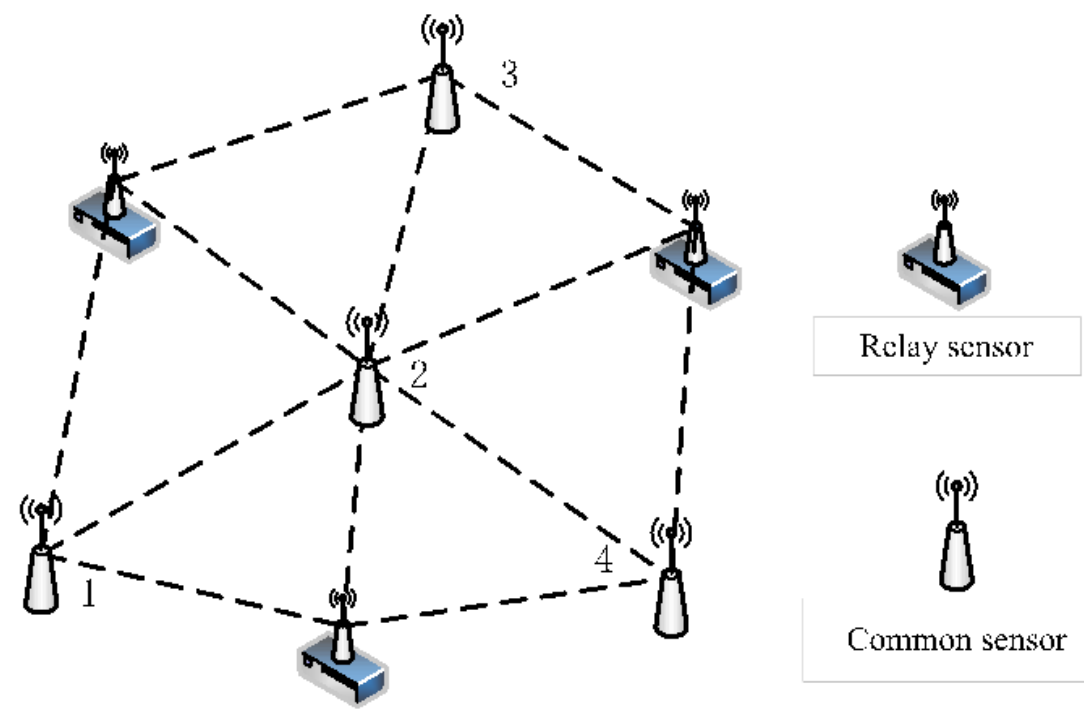

Fig. 3. The network reconfiguration design scheme 


\section{Robust opportunistic guarantee architecture and implementation}

\subsection{Architecture}

The overall design of the system is shown in Figure 4. The lower level of the system is composed of sensor nodes (Sensor Node), relay nodes (Repeater) and sink nodes (Sink Node). Each node has the early fault warning mechanism to increase survivability. The transport layer use the WiFi (Wireless Fidelity) protocol for transmission. The data processing, data storage and package unpacking mining progress would be completed in Host computer.

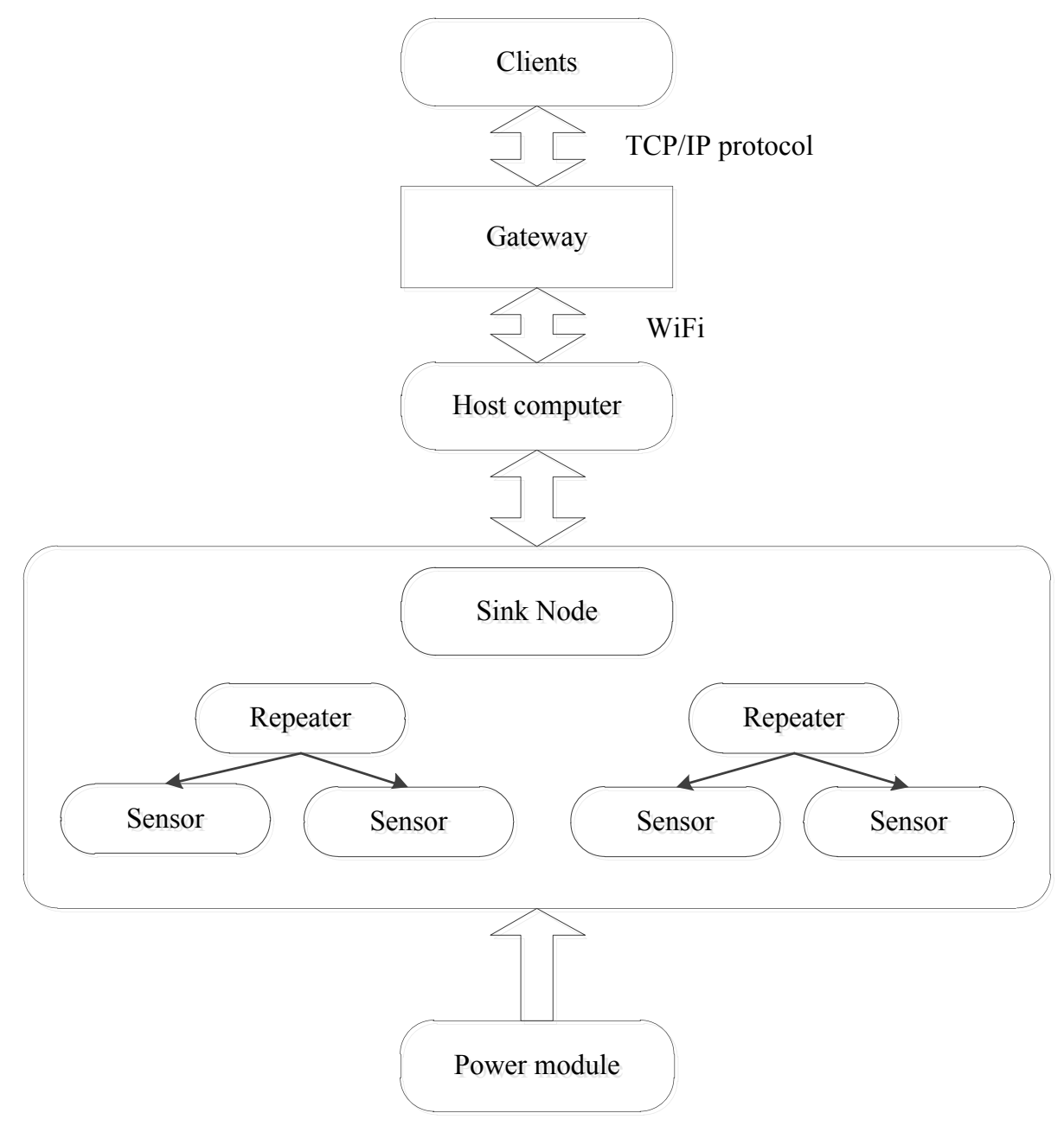

Fig. 4. The Overall system design scheme 
The indoor environmental monitoring system is the development foundation, in order to improve the survivability ability of WSNs environment monitoring system. The system used the real-time data transmission of sensor nodes with WiFi protocol. The information collected by sensor nodes mainly include the environment temperature and humidity, $\mathrm{CO}, \mathrm{CO}_{2}$, light, air quality, air pressure, smoke, vibration and inclination and the intensity of ultraviolet light. The whole system model is shown in Figure 5. This system prolong the network communication distance, reduce the power consumption of ordinary nodes and the node failure rate, and ultimately improve the system survivability ability through relay nodes.

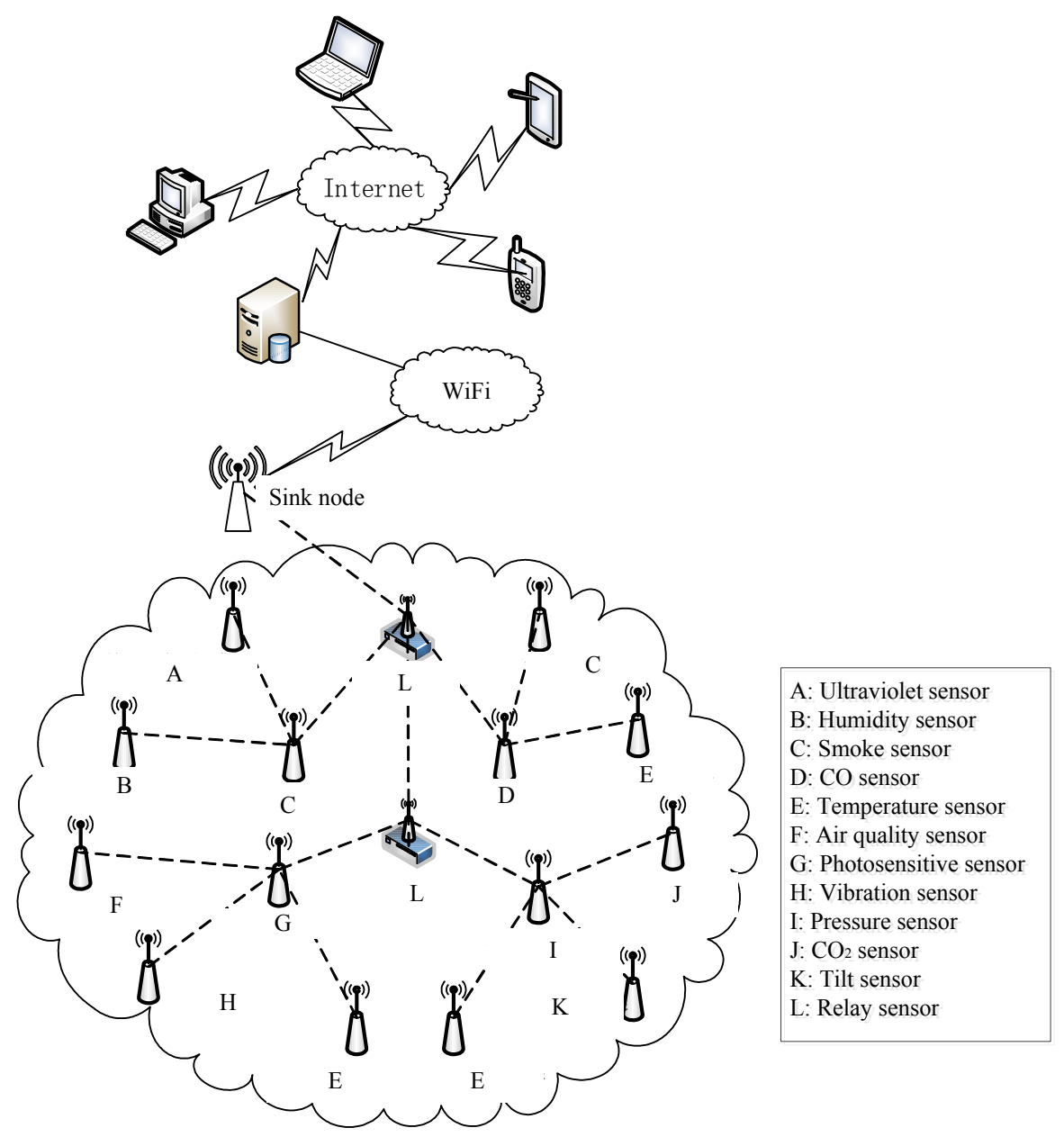

Fig. 5. The whole system model 


\subsection{Implementation}

The system uses the modular design method, including data acquisition module, data transmission module, data storage module, power supply module, and data display module.

Data acquisition process is shown in Figure 6. The initialization operation considers the timer initialization, serial initialization and sensor initialization. When the timer interrupt subroutine ReadFlag is 1 , the initialization work is completed. And then data acquisition, data processing and data transmission would be considered. However, when the ReadFlag is 0 , the initialization of sensor fails. It is necessary to re initialize the sensor operation, until the initialization of the sensor can be successfully carried out a series of data acquisition operations.

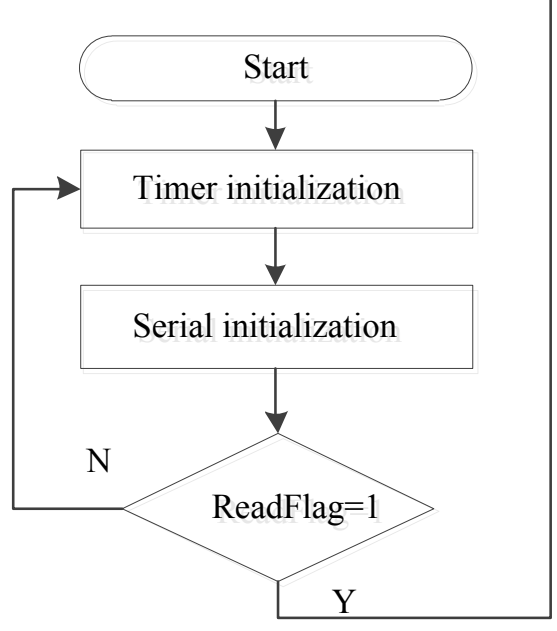

Y

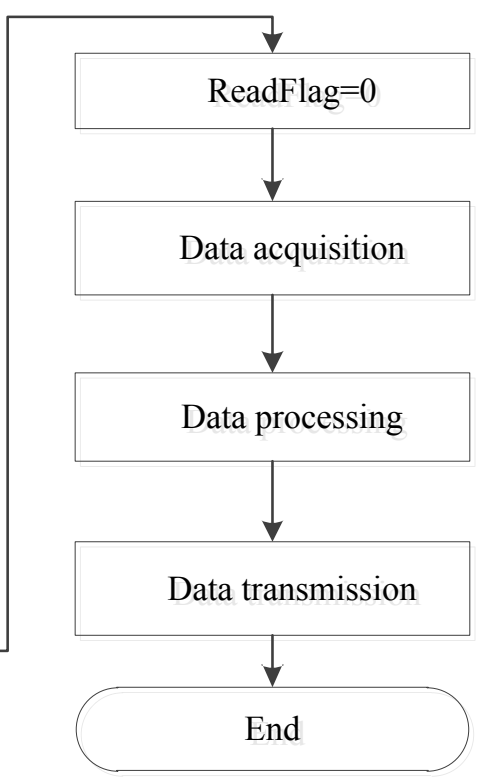

Fig. 6. Data acquisition process

Host computer fault polling detection procedures is proposed. Here, we show the temperature sensor node as an example to describe the threshold. When the temperature and humidity of the node is not in the range of the threshold, the speaker alarm, when the sensor does not collect any information that the node failure, Led lights would be flashing. Data collected by sensors would be sent to MCU (Microcontroller Unit) for processing through the serial port, the WiFi module, and wireless transmission. The data packet contains the region number (Area ID), node number (Node ID), (Sensor Type) type sensor data acquisition date (Sensor Data) the data cutoff mark (End). The packet format is shown in Table 1. 
Table 1. data packet format

\begin{tabular}{|l|l|l|l|l|}
\hline \multicolumn{1}{|c|}{ Area Flag } & \multicolumn{1}{|c|}{ Node Flag } & Sensor Type Flag & Sensor Data Flag & \multicolumn{1}{c|}{ End Flag } \\
\hline Area ID & Node ID & Sensor Type & Sensor Data & End \\
\hline
\end{tabular}

\section{Performance evaluation}

In this paper, the location of sensor nodes is based on the environment of Changshu museum. The sensor node distribution is shown in Figure 7. The black point represents the common perception node. There are many exhibition halls in Museum. The distance between the exhibition halls is large. In this experimental environment, we evaluated the proposed opportunistic cooperative sensing robust guarantee mechanism denoted as OCSRG and the original scheme for Indoor complex environment monitoring. In the network communication, we must increase the relay nodes to extend the network communication distance, improve the communication quality of the network and prolong the life cycle of the node. Thus, even if the energy of sensor nodes is reduced, the communication between nodes can still be normal.

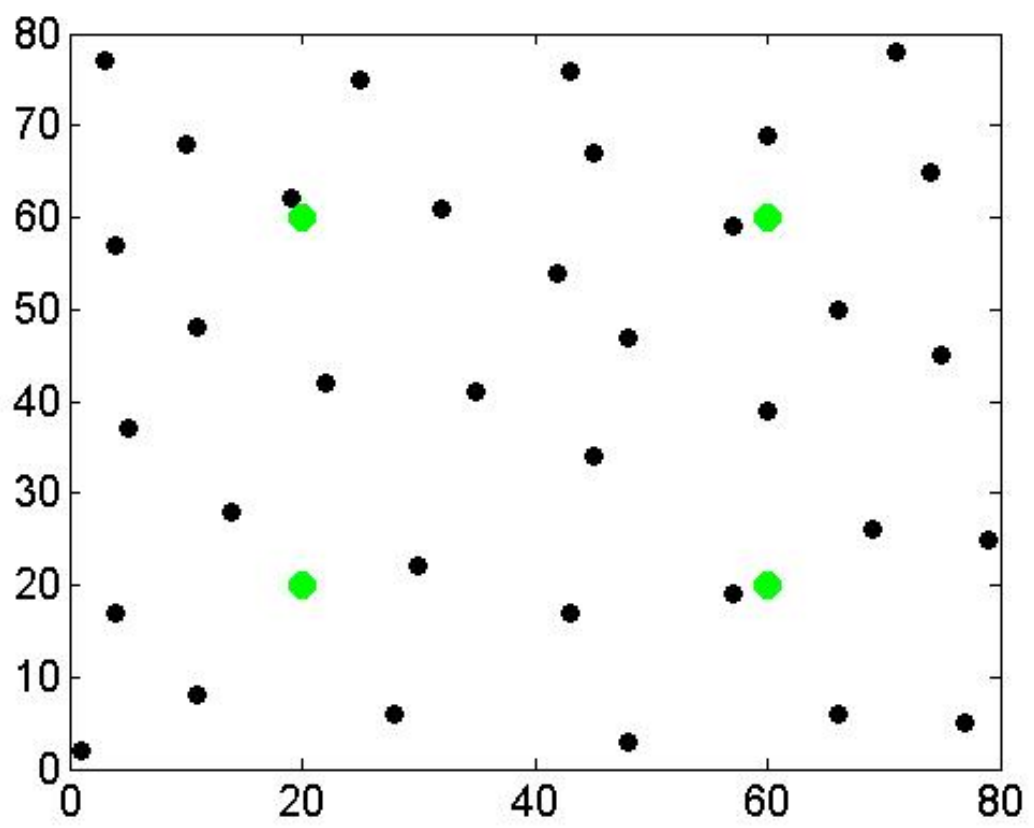

Fig. 7. Sensor node distribution

The survivability evaluation is tested the system communication distance and the system life cycle. This system theory would be completed and evaluated through redundancy mechanism and network reconfiguration model improvement strategies to improve the survivability of the communication link. In the hardware design, the network reconfiguration would be certificated, which could improve the survivability 
of the system by increasing the relay node, data polling query mechanism and hardware design.

As shown in Figure 8, the communication distance test and comparison of the data field, we can see that the hardware design of the improved network communication distance is greatly improved. So, the long-distance environment monitoring sensor nodes communication between different halls can be maintained in the museum and the quality of service can be guaranteed. In addition, due to the increase of the relay nodes, the sensing nodes of the bottom layer will reduce energy consumption, the life cycle of sensor nodes will be extended, and network resilience would be enhanced. So, the system would be more stable and efficient.

The hardware design scheme of the system not only increases the communication distance of the network, but also reduces the workload of the remote data transmission in the network cluster. So the sensors energy consumption will be reduced, so the life cycle of the sensor node is also extended. Figure 9 shows the electricity use of the 1800 mAh lithium battery node monitoring comparison chart. Figure 10 gives the electricity use of the comparison of $2500 \mathrm{mAh}$ lithium battery monitoring node. Through comparative analysis of the data, we found that the relay node can prolong the life cycle of the system at the same time. The use of high performance and high capacity battery can also effectively improve the survivability of the system.

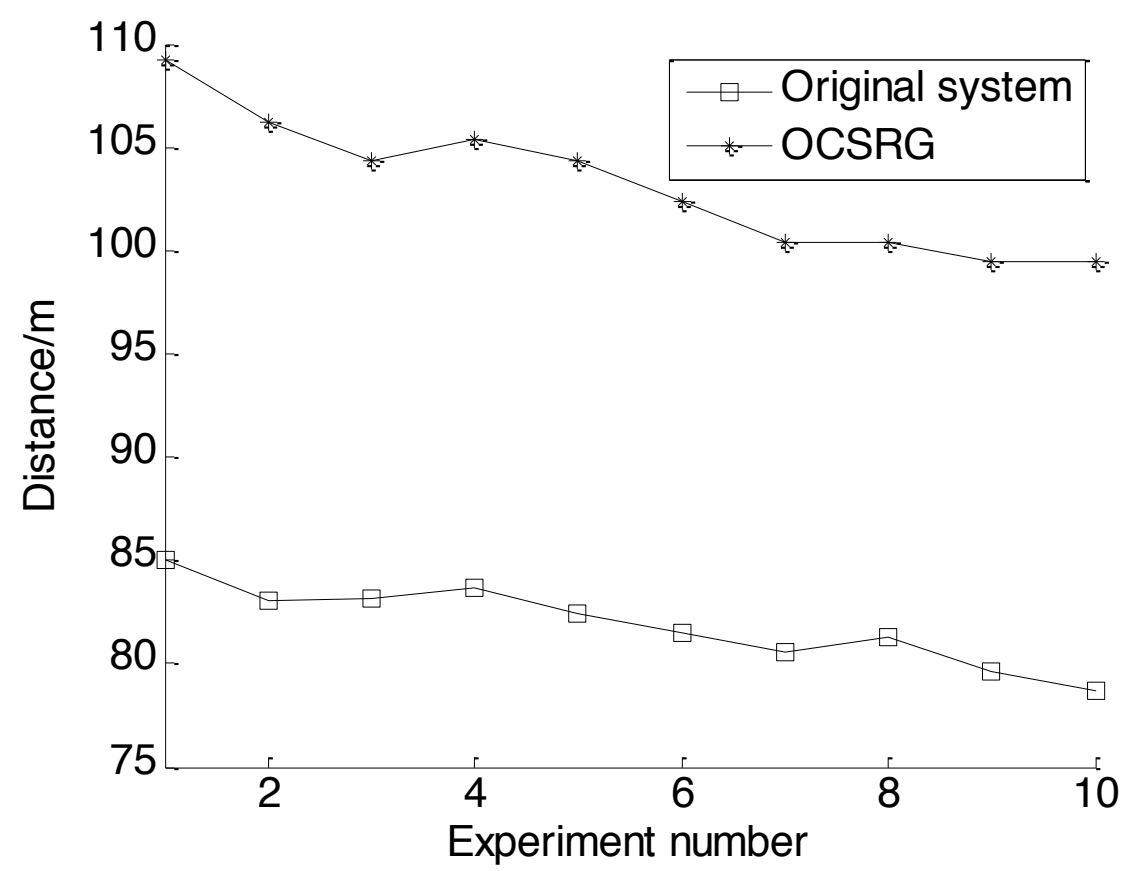

Fig. 8. Sensor node distribution 


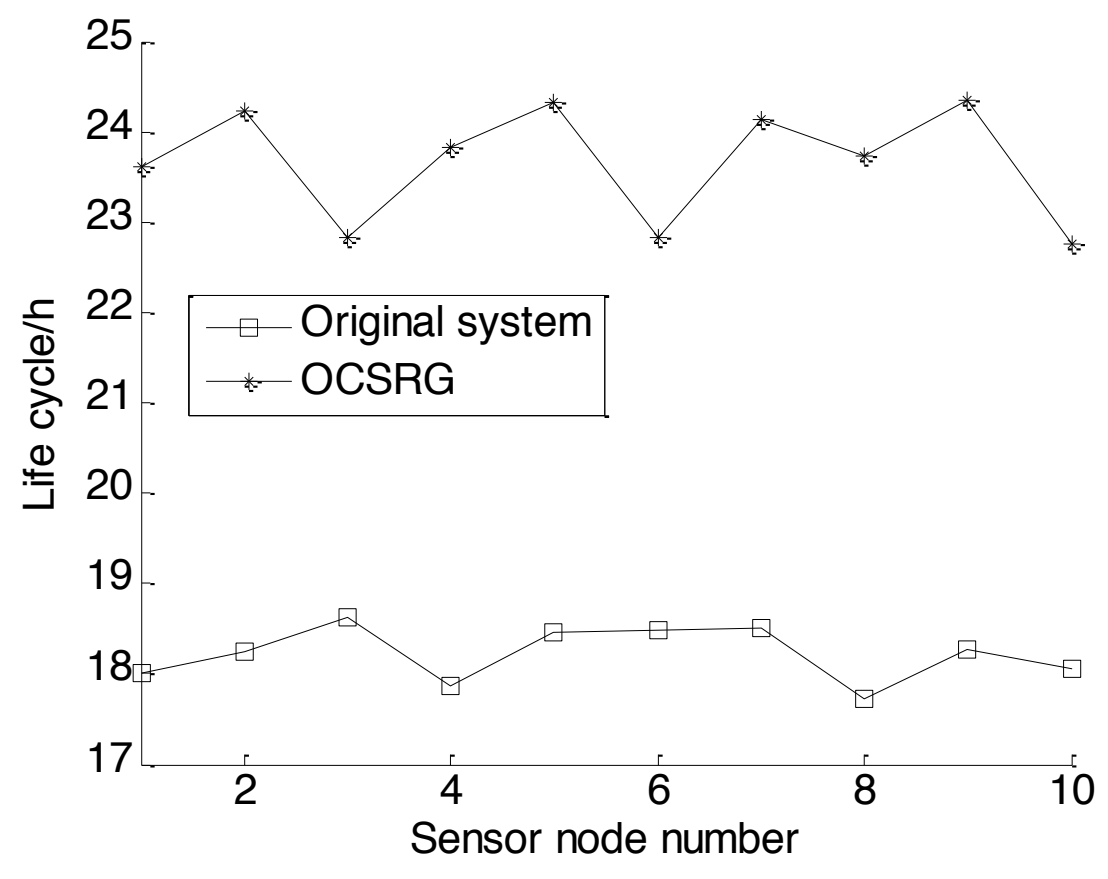

Fig. 9. $1800 \mathrm{mAh}$ battery monitoring

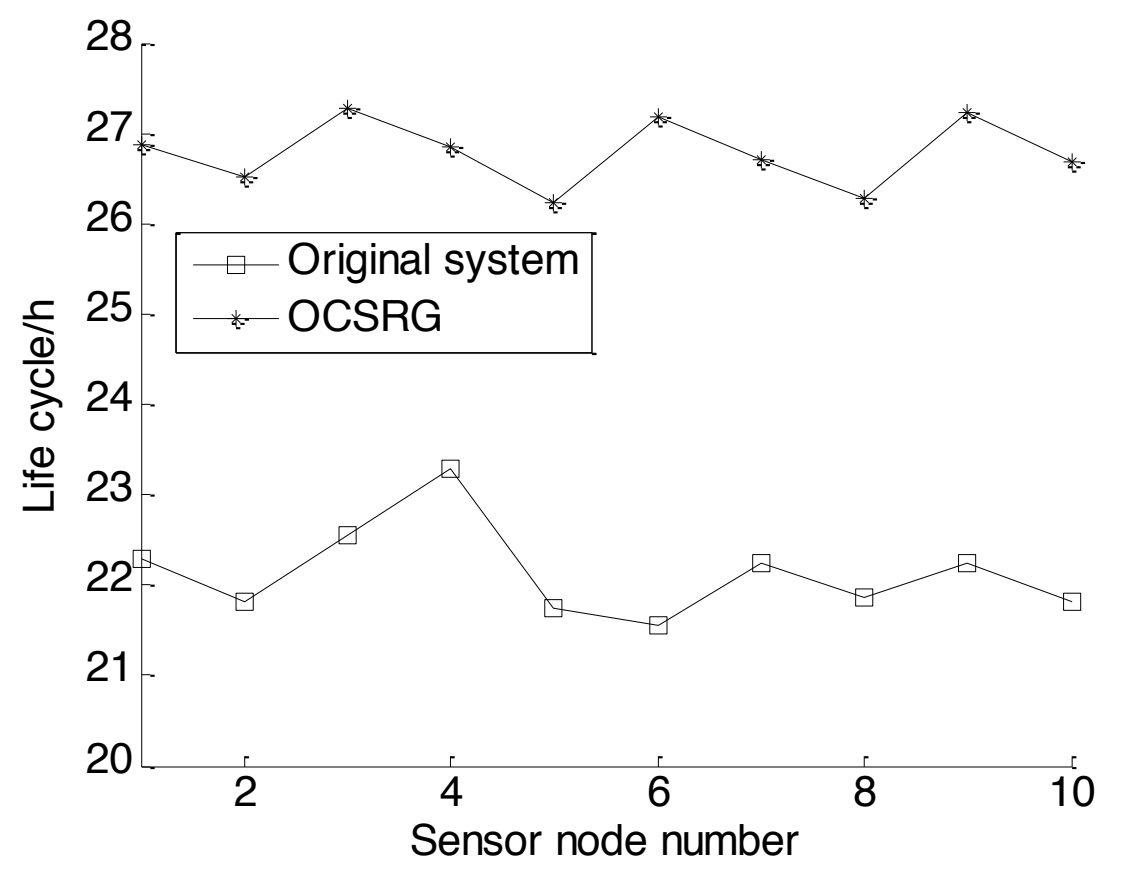

Fig. 10. $2500 \mathrm{mAh}$ battery monitoring 


\section{Conclusions}

The software optimization from the existing cluster head redundancy and link redundancy algorithm model, we fused the above algorithms. About the hardware optimization, we mainly considered the network reconfiguration with relay nodes, through the fault polling to check whether abnormal environment. The communication distance would be extended and the energy consumption of sensor nodes could be reduced, the life cycle of a system could be prolonged by the proposed opportunistic cooperative sensing robust guarantee mechanism. Through the field test experiments, we found that the system survivability and robust ability with the proposed scheme has been significantly improved.

\section{Acknowledgment}

This work is supported in part by the Science and technology project of Jiangsu Provincial Archives Bureau (No. 2016-14), the Scientific research project of Jiangsu Provincial Department of culture (No. 16YB12), the Suzhou 2016 annual industrial application research (industry) guidance program, the Suzhou science and technology development project (NO. SYSD2015014), the Natural Science Foundation of China under grant No. 61402057, the Natural Science Foundation of Jiangsu Province under grant No. BK20140418, the National Natural Science Foundation of China NO. 61602062 and the Natural Science Foundation of Jiangsu Province NO. BK20160410.

\section{$7 \quad$ References}

[1] Lee J Y, Seok J H, Lee J J (2012). Multiobjective Optimization Approach for Sensor Arrangement in A Complex Indoor Environment. IEEE Transactions on Systems Man \& Cybernetics Part C, 42(2): 174-186. https://doi.org/10.1109/TSMCC.2010.2103310

[2] Bamba A, Joseph W, Vermeeren G, et al. (2013). Validation of experimental whole-body SAR assessment method in a complex indoor environment. Bioelectromagnetics, 34(2): 122-132. https://doi.org/10.1002/bem.21749

[3] Van Schaik P, Mayouf M, Aranyi G. (2015). 3-D route-planning support for navigation in a complex indoor environment. Behaviour \& Information Technology, 34(7): 713-724. https://doi.org/10.1080/0144929X.2015.1004649

[4] Kropat G, Bochud F, Jaboyedoff M, et al. (2015). Predictive analysis and mapping of indoor radon concentrations in a complex environment using kernel estimation: An application to Switzerland. Science of the Total Environment, 505: 137-148. https://doi.org/10.1016/j.scitotenv.2014.09.064

[5] Zeilinger M N, Morari M, Jones C N. (2014). Soft Constrained Model Predictive Control With Robust Stability Guarantees. IEEE Transactions on Automatic Control. 59(5): 11901202. https://doi.org/10.1109/TAC.2014.2304371

[6] Zarei J, Shokri E. (2014). Robust sensor fault detection based on nonlinear unknown input observer, Measurement, 48(2): 355-367. https://doi.org/10.1016/j.measurement. $\underline{2013.11 .015}$ 
Paper-Opportunistic Cooperative Sensing for Indoor Complex Environment Monitoring

[7] Esfahani P M, Kuhn D. (2015). Data-driven Distributionally Robust Optimization Using the Wasserstein Metric: Performance Guarantees and Tractable Reformulations. Mathematics, 24(9): 711-712.

[8] Zhang X, Liu W, Xu Y, et al. (2014). Quaternion-valued robust adaptive beamformer for electromagnetic vector-sensor arrays with worst-case constraint. Signal Processing, 104(6): 274-283. https://doi.org/10.1016/j.sigpro.2014.04.006

[9] Li B, Cui W, Wang B. (2015). A Robust Wireless Sensor Network Localization Algorithm in Mixed LOS/NLOS Scenario. Sensors, 15(9): 23536-23553. https://doi.org/10.3390/ $\underline{\mathrm{s} 150923536}$

[10] Lin F T, Kuo Y C, Hsieh J C, et al. (2015). A Self-Powering Wireless Environment Monitoring System Using Soil Energy. IEEE Sensors Journal, 15(7): 1-1. https://doi.org/10.1109/JSEN.2015.2398845

[11] Bonamano S, Piermattei V, Madonia A, et al. (2016). The Civitavecchia Coastal Environment Monitoring System (C-CEMS): a new tool to analyze the conflicts between coastal pressures and sensitivity areas. Ocean Science, 12(1): 87-100. https://doi.org/10.5194/os$12-87-2016$

[12] 12 Zhen-Ning D U, Amp Y V.(2015) Implementation Method of the Greenhouse Environment Monitoring System Based on Wireless Sensor. Journal of Oral Biosciences, 25(8): 286-292.

[13] S. H. Lee(2014), Study for overseas expansion of the internet of things. DeltaTech International: Korea Communications Policy Research, 14(35): 39.

[14] Ossama Younis, Sonia Fahmy, Paolo Santi(2010). An Architecture for Robust Sensor Network Communications. International Journal of Distributed Sensor Networks, 1: 305327. https://doi.org/10.1080/15501320500330786

[15] Han X, Cao X, Lloyd E L, et al(2010). Fault-Tolerant Relay Node Placement in Heterogeneous Wireless Sensor Networks. IEEE Transactions on Mobile Computing, 9(5): 643656. https://doi.org/10.1109/TMC.2009.161

\section{Authors}

Yong Jin (corresponding author) was born in 1981, and received a master of engineering (2009) in Computer Science and Technology from the Nanjing University of Technology. Since 2009, he has been working at the School of Computer Science and Endineering, Changshu Institute of Technology, Changshu, 215500, China, as a lecturer in School of Computer Science and Engineering. His research interests are in mobile ad hoc networks, multimedia communication, cooperative communication, wireless sensor networks, QoS, performance evaluation, error control, etc. (e-mail: jinyong@cslg.cn).

QIAN Zhenjiang was born in 1982, Ph.D., associate professor. He is with the School of Computer Science and Engineering, Changshu Institute of Technology, Changshu, 215500, China. His current research interests focus on operating system security, formal verification and embedded system.

Xiaoshuang Xing received her PhD degree in Communication and Information System from Beijing Jiaotong University, China, in 2014. She is an Associate Professor in the School of Computer Science and Engineering, Changshu Institute of Technology, Changshu, 215500, China. Her primary research interests include: spectrum 
prediction in cognitive radio networks, resource management, VANET, and mobile social networks.

Lu Shen are undergraduate students in School of Computer Science and Engineering, Changshu Institute of Technology, Changshu, 215500, China. Their research interests include wireless sensor networks, Internet of Things.

Article submitted 31 January 2017. Published as resubmitted by the authors 15 March 2017. 